\title{
Caracterización de consumidores agroindustriales de carne de pequeños rumiantes en el Estado de México
}

\author{
Characterization of agroindustrial consumers of small \\ ruminants meat in the State of Mexico
}

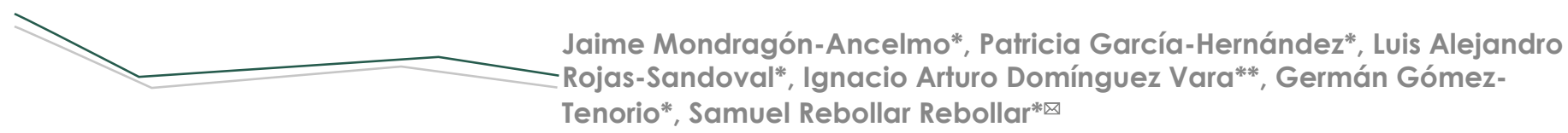

Mondragón-Ancelmo, J., García-Hernández, P., Rojas-Sandoval, L. A., Domínguez Vara, I. A., Gómez-Tenorio, G., \& Rebollar Rebollar, S. (2018). Caracterización de consumidores agroindustriales de carne de pequeños rumiantes en el Estado de México. Investigación y Ciencia de la Universidad Autónoma de Aguascalientes, 26(74), 17-24.

Se caracterizó a los consumidores agroindustriales (CAl) de pequeños rumiantes para carne (PRC) en el Estado de México. Con una encuesta semiestructurada se obtuvo información sociodemográfica, de comercialización e importancia de los atributos de los PRC (peso vivo, sexo, raza, entre otros). Los datos se analizaron con la técnica de componentes principales, prueba de Tukey y Kruskal-Wallis. De acuerdo con su similitud entre objetos $(73.3 \%$ de la varianza total y $\mathrm{KMO}=0.802$ ), se obtuvieron tres grupos de CAl: Grupo 1. Barbacoyero urbano, Grupo 2. Barbacoyero/birriero rural, y Grupo 3. Carnicero. Los Grupos 1 y 3 adquirieron animales de menor peso ( $p$ $<0.05$ ) y el Grupo 3 pagó $7 \%$ más del precio de los animales con respecto a los grupos 1 y $2(p<0.05)$. Se evidenció que existen tres grupos de CAl que difieren en sus características y exigencias de los atributos de calidad de los PRC.

Palabras clave: carne; ovinos; caprino; análisis de componentes principales; barbacoyero; birriero.

Keywords: meat; sheep; goats; principal component analysis; barbacoyero; birriero.

\section{Recibido: 18 de octubre de 2017, aceptado: 5 de marzo de 2018}

* Centro Universitario Temascaltepec, Universidad Autónoma del Estado de México. Carretera Toluca-Tejupilco km. 67.5, Barrio de Santiago, C. P. 51300, Temascaltepec de González, México. Correo electrónico: jaimemond_01@hotmail.com;garciahernandezp@yahoo.com.mx lars23x@gmail.com; gomte61@yahoo.com.mx; samrere@hotmail.com

** Facultad de Medicina Veterinaria y Zootecnia, Universidad Autónoma del Estado de México. Campus universitario El Cerrillo, C. P. $\mathbf{5 0 0 9 0}$ Toluca, México. Correo electrónico: igy92@hotmail.com

$\otimes \quad$ Autor para correspondencia
The agroindustrial consumers (AIC) of small ruminants for meat (SRM) were characterized in the State of Mexico. With a semi-structured survey, sociodemographic information, about commercialization and importance of the attributes of the SRM (live weight, sex, race, among others) was obtained. The data were analyzed with the technique of principal components, Tukey test and Kruskal-Wallis. According to their similarity between objects $\mathbf{7 3 . 3 \%}$ of the total variance and $\mathrm{KMO}=0.802$ ), three groups of CAI were obtained: Group 1. Urban barbacoyero, Group 2. Rural barbacoyero/birriero, and Group 3. Butcher. Groups 1 and 3, acquired animals of lower weight ( $p$ $<0.05$ ) and Group 3, paid $7 \%$ more than the price of animals with respect to groups 1 and 2 ( $p<0.05)$. It was evidenced that there are three groups of AIC that differ in their characteristics and demands of the quality attributes of the SRM.

\section{INTRODUCCIÓN}

En México, la producción de carne de ovinos y caprinos participa con $4 \%$ de la carne que se produce en el país (SIAP, s. f.), esto se traduce en una relevancia económica y social. Se trata de un sector heterogéneo, en el que conviven diferentes grados de desarrollo y escenarios de cría, que van desde producción familiar hasta producción tecnificada e industrial (Galaviz-Rodríguez et al., 2011; Juárez-Morales et al., 2012; Nuncio-Ochoa, Nahed, Díaz Hernández, Escobedo Amezcua, \& Salvatierra Izaba, 2001). Lo anterior favorece una variación en los atributos 
IIVESTIGACIÓn Y CIERCIA DE LA UחIVERSIDAD AUTÓNOTH DE AGUASCALIERTES intrínsecos y extrínsecos de la carne que influyen en la aceptación del producto por el consumidor (Fonti-Furnols \& Guerrero, 2014), aunado a los patrones de consumo, como diversificación en el estilo de vida del consumidor (Bernués, Ripoll, \& Panea, 2012), influencia de factores geográficos, culturales, climáticos y económicos (Nam, Jo, \& Lee, 2010).

Los procesos de producción, industrialización, comercialización, preparación y consumo de alimentos se han convertido en factores determinantes de la calidad de los productos cárnicos que se ofrecen al consumidor final ( $\mathrm{Nam}$ et al., 2010). Los pequeños rumiantes para carne (PRC) recorren una larga cadena de producción para llegar al plato: desde el productor de animales hasta el consumidor final (Mondragón-Ancelmo et al., 2014; Rebollar et al., 2007). En cada uno de los eslabones de producción de esta cadena se consideran diferentes atributos de calidad intrínseca como son los aspectos físicos: raza, sexo, color, tamaño, peso, salud, apariencia de la carne, etc., y extrínseca, como son los aspectos de producción y/o elaboración: marca, sello de calidad, origen, embalaje, información de producción, bienestar animal, entre otros (Bernués, Olaizola, \& Corcoran, 2003).

El Estado de México es un caso representativo, en esta zona se comercializan ovinos y caprinos para carne que son de producción local o de otros estados del país (Mondragón-Ancelmo et al., 2014; Rebollar et al., 2007). El consumo de carne de pequeños rumiantes oscila entre los 400 y los 700 g/año (SIAP, s. f.). La mayor parte de esta carne se consume en forma de barbacoa/birria' y en los últimos años los consumidores agroindustriales (CAI) han incursionado en la elaboración de cortes de carne de alto valor, debido al conocimiento desarrollado a partir de las necesidades de sus clientes. En consecuencia, los CAl tienen exigencias particulares sobre los atributos de calidad que deben reunir los animales in vivo. Por tanto, el objetivo de este estudio fue determinar las características de los consumidores agroindustriales de ovinos y caprinos para carne en el Estado de México.

1 Barbacoa: producto obtenido de la carne cocinada en hoyo u horno precalentado. Los insumos básicos son penca de maguey, ajo, cebolla, chile, agua y carne de ovino. Birria: producto obtenido de la carne cocinada en perol. Los insumos básicos son salsa de chile, agua y carne de caprino. Los ingredientes y el proceso de preparación dependerán de cada cocinero y de las costumbres de cada región.
MATERIALES Y MÉTODOS

El estudio se realizó en 2015 en la zona sur, norte, este y poniente del Estado de México; entidad reconocida como la primera en acopio, transformación y comercialización de PRC a nivel nacional (comunicación personal: Raúl Conde García, presidente de la Asociación de Barbacoyeros de Capulhuac, Estado de México). Se realizó un muestreo por intención a 67 propietarios de los establecimientos que comercializan carne en forma de barbacoa, birria, canales o cortes (Cochran, 1984).

A partir de la aplicación de una encuesta semiestructurada a 67 propietarios se obtuvo información sociodemográfica (edad, escolaridad, años en la actividad y tipo de agroindustria) y de comercialización (peso y precio de compra de los animales en pie, venta y precio del producto procesado al consumidor final). También se reunió información acerca de la importancia de los atributos de ovinos y caprinos in vivo como especie, peso vivo, sexo, raza, edad, salud, alimentación, sin anabólicos, precio, bienestar animal y procedencia (Bernués et al., 2003; Bernués et al., 2012) a través de una escala actitudinal de Likert con opciones de respuesta (1. Nada importante a 5. Muy importante) (Kinnear \& Taylor, 1993).

La información de las encuestas se analizó con el software IBM SPSS Statistics versión 22. Se utilizaron técnicas de análisis multivariado factorial y de conglomerados. El análisis factorial se aplicó a las preguntas relacionadas con el nivel de importancia de los atributos de tipo ordinal; en esta técnica pueden obviarse los supuestos de linealidad y normalidad en las variables mientras se cuente con un mínimo de 50 observaciones (Hair, Anderson, Tatham, \& Black, 1999). El método de componentes principales se utilizó para extraer factores y el índice de Kaiser-MeyerOlkin (KMO) para medir la correlación entre las variables; $\mathrm{KMO}: 1=$ alta,$\geq 0.9=$ buena,$\geq 0.8=$ notable,$\geq$ $0.7=$ mediana,$\geq 0.6=$ baja $y<0.5=$ muy bajo. La prueba de esfericidad de Bartlett se utilizó para evaluar la aplicabilidad del análisis factorial de las variables estudiadas: Si Sig. (p-valor) < 0.05 (se acepta hipótesis nula), se puede aplicar el análisis factorial. Si Sig. (p-valor) > 0.05 (se rechaza la hipótesis nula), no se puede aplicar el análisis factorial (Hair et al., 1999).

Los factores seleccionados fueron los que presentaron valores propios $\geq 1$. Con el fin de obtener 


\section{IIVESTIGAGIÓn Y CUERCIA DE LA UחIVERSIDAD AUTÓNOMA DE AGUASCALIERTES}

una mejor comprensión de los factores se aplicó una rotación ortogonal Varimax, es la más utilizada, la cual consigue que cada componente rotado presente correlaciones solamente con unas cuantas variables, y es adecuada cuando el número de componentes es reducido. Los puntajes de los factores se estimaron por el método de regresión y se utilizaron como nuevas variables para el análisis de conglomerados (Hair et al., 1999).

Para identificar perfiles de CAI de acuerdo con las actitudes de importancia hacia los atributos de los animales (Hair et al., 1999), se realizó un análisis de conglomerados jerárquicos con el método de Ward con medida de similitud entre objetos. Los grupos obtenidos se definieron por sus características sociodemográficas y de comercialización. Finalmente se aplicaron técnicas de análisis bivariado para observar posibles cambios significativos entre las variables de muestras independientes: prueba de Tukey para las variables sociodemográficas y de comercialización, y Kruskal-Wallis para la variable de atributos de los animales (Daniel, 2005; Hair et al., 1999).

\section{RESULTADOS}

A partir del análisis factorial se obtuvieron tres factores que explicaron $73.3 \%$ de la varianza total. La prueba de esfericidad de Bartlett $(p<0.001)$ y $\mathrm{KMO}=$ 0.802 indican que las variables incluidas en el análi- sis están significativamente correlacionadas entre sí (tabla 1). El primer factor, denominado atributos intrínsecos, relaciona las variables de peso, sexo, raza y edad de los animales y bienestar animal, atributos que contribuyen a lograr mayor calidad del producto destinado a consumidores finales exigentes. El segundo factor, denominado atributos extrínsecos, incluye factores como el respeto por el bienestar de los animales, así como la preferencia por la no adición de anabólicos en la alimentación de los animales, práctica que genera desconfianza y posibles daños a la salud del consumidor final. Entre estos atributos extrínsecos también destacó la preferencia por precios bajos como decisor de compra. El tercer factor se denominó especie, y está relacionado con la preferencia que el CAl tiene por ciertas especies, de acuerdo con el destino final de la carne (birria, barbacoa, canales o cortes). Estos resultados ponen en evidencia que de acuerdo con las actitudes de los CAl, los sistemas de producción de PRC deberán buscar esquemas de diferenciación del producto enfocados en atributos intrínsecos, extrínsecos y especie animal.

El análisis de clúster realizado con variables de distancia de las puntuaciones de factores de los CAl con respecto a los tres factores relacionados con las actitudes sugiere que hay tres grupos de CAl: Grupo 1, barbacoyero urbano 31 (46\%), Grupo 2, barcoyero/birriero rural 26 (39\%) y Grupo 3, carnicero 10 (15\%) (tabla 2).

Tabla 1

Matriz de factores rotados del consumidor agroindustrial de pequeños rumiantes para carne

\begin{tabular}{lrrr}
\hline \multirow{2}{*}{ Atributos del animal } & \multicolumn{3}{c}{ Componentes } \\
\cline { 2 - 4 } Peso & Factor 1 & Factor 2 & Factor 3 \\
Sexo & $\mathbf{0 . 9 3 5}$ & 0.075 & -0.033 \\
Raza & $\mathbf{0 . 9 1 5}$ & 0.042 & 0.030 \\
Edad & $\mathbf{0 . 8 8 4}$ & 0.126 & -0.053 \\
Procedencia & $\mathbf{0 . 7 7 6}$ & 0.271 & -0.191 \\
Sin anabólicos & -0.384 & $\mathbf{0 . 7 9 7}$ & 0.246 \\
Precio & 0.286 & $\mathbf{0 . 6 9 1}$ & -0.068 \\
Alimentación & -0.291 & $-\mathbf{0 . 6 4 8}$ & -0.191 \\
Bienestar animal & 0.072 & $\mathbf{0 . 6 2 6}$ & $-\mathbf{0 . 5 0 9}$ \\
Especie & $\mathbf{0 . 5 5 8}$ & $\mathbf{0 . 5 9 6}$ & 0.178 \\
\hline Variancia por factor (\%) & -0.059 & 0.187 & $\mathbf{0 . 8 7 3}$ \\
\hline Varianza acumulada (\%) & 41.74 & 20.51 & 11.13 \\
\hline Nota: Elaborán propia & 41.74 & 62.25 & $\mathbf{7 3 . 3 7}$ \\
\hline
\end{tabular}

Nota: Elaboración propia. 
Las características sociodemográficas y de comercialización de los grupos de CAl se indican en la tabla 2. El Grupo 1 elabora y comercializa barbacoa de carne de ovino o caprino, o combinación de carne de ambas especies; estos CAl se encuentran principalmente en las rutas, vías públicas, mercados y en restaurantes ubicados en las entradas o dentro de las principales ciudades del Estado de México. Asisten a posibles consumidores finales con mayor poder adquisitivo y exigencia en la calidad del servicio. El Grupo 2 son CAI tradicionales de barbacoa (elaborada con carne de ovinos) o birria (elaborada con carne de caprinos), se encuentran principalmente en los pueblos o áreas rurales, y comercializan el producto directamente al consumidor final que acude en las rutas, en las vías públicas o mercados de los pueblos.

Los grupos 1 y 2 son CAl con mayor experiencia en la industria cárnica de la barbacoa o birria ( $p$ $<0.004)$, asignaron el precio más alto de venta de carne procesada al consumidor final ( $p<0.001)$, se debe a que la carne procesada en barbacoa o birria se convierte en un platillo típico de los más caros de la carne ovina y caprina en México, asociado al conocimiento del proceso artesanal que da mayor valor agregado en el comercio del producto cárnico. El Grupo 1 prefiere menor contenido de grasa de la canal de ovinos o caprinos para elaborar barbacoa o birria; por tanto, adquirió animales de menor peso $(p<0.001)$.

El Grupo 3 se ubica en ciudades estratégicas para procesar y comercializar la carne de ovino o caprino. Se caracterizó por agrupar CAI con mayor año de escolaridad ( $p<0.002$ ), estos tienen una actitud bastante positiva para mejorar la calidad de sus productos continuamente, así que han incursionado en la venta de cortes de carne ovino o caprino, y tienen mayores ventas de carne en canal o cortes por semana ( $p<0.001)$, son proveedores de la industria de la barbacoa, birria, restaurantes y consumidor final (figural). Estos pagaron 7\% más del precio de compra al proveedor de animales $(p<0.001)$.

Con base en el análisis de Kruskal-Wallis (figura 2 ) hubo diferencias estadísticas ( $p<0.001$ ) entre grupos de CAl sobre los atributos de los animales, excepto en salud ( $p>0.438$ ) (figura 2). Los grupos 1 y 3 dieron mayor importancia a los animales machos de razas mejoradas, menor $a \leq 1$ año de edad y pesos que oscilan de 40.7 a $45.5 \mathrm{~kg}$. En el Grupo 3 prefirieron animales alimentados con base en concentrado

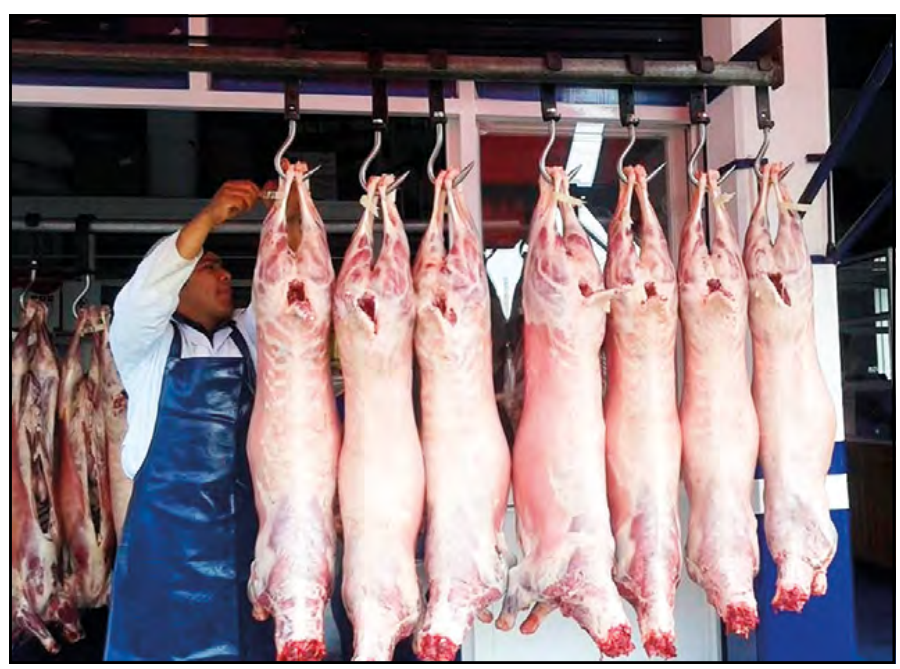

Figura 1. Carnicería (venta en canal y cortes de carne) de Capulhuac, Estado de México.

Fotografía de Jaime Mondragón-Ancelmo.

y bajo condiciones favorables de bienestar y salud animal para que el producto pueda competir en el mercado de venta de canal o cortes. El Grupo 2 dio mayor importancia a los animales no alimentados con anabólicos (hormonas, ßeta adrenérgicos, etc.).

La especie animal es importante; para quienes elaboran barbacoa necesariamente debe ser carne de ovino, y para quienes elaboran birria debe ser de caprino. El precio resultó ser más importante para los Grupos 1 y 2, estos buscan abaratar los costos de producción en la elaboración de la barbacoa o birria; por tanto, compran animales de menor precio; no así para el Grupo 3, que puede mejorar e incentivar el precio de un animal de mayor calidad.

\section{DISCUSIÓN}

En estudios previos se ha encontrado que ciertos mercados de pequeños rumiantes privilegian atributos intrínsecos, como la raza, el peso, género, edad y color pelo o lana (Kassa, Haile, \& Essa, 2011). Estos resultados son consistentes con los encontrados en este estudio, donde algunos de los CAl encuestados privilegiaron estos atributos. Estas preferencias están en función de las exigencias de los diferentes procesos de producción a los que se destina la carne adquirida (barbacoa, birria, canales o cortes), así como de las necesidades del consumidor final de estos productos.

Por ejemplo, existe una tendencia en aumento entre los consumidores que buscan platillos elabora- 
Tabla 2

Perfil demográfico y de comercialización de los tres grupos de consumidores agroindustriales de pequeños rumiantes de carne

\begin{tabular}{|c|c|c|c|c|c|}
\hline Variables & $\begin{array}{c}\text { Grupo } 1 \\
(n=31)\end{array}$ & $\begin{array}{c}\text { Grupo } 2 \\
(n=26)\end{array}$ & $\begin{array}{c}\text { Grupo } 3 \\
(n=10)\end{array}$ & Total & $p$ \\
\hline \multicolumn{6}{|l|}{ Sociodemográficas } \\
\hline \multicolumn{6}{|l|}{ Tipo de consumidor agroindustrial } \\
\hline Barbacoyero & 31 & 17 & - & 48 & - \\
\hline Birriero & - & 9 & - & 9 & - \\
\hline Carnicero & - & - & 10 & 10 & - \\
\hline \multicolumn{6}{|l|}{ Sexo del consumidor agroindustrial } \\
\hline Masculino, \% & 81.0 & 96.0 & 96.0 & - & - \\
\hline Femenino, \% & 19.0 & 4.0 & 4.0 & - & - \\
\hline \multicolumn{6}{|l|}{ Experiencia en la actividad } \\
\hline Edad, años & 43.8 & 42.7 & 34.8 & 42.0 & 0.128 \\
\hline Estudios, años & $9.3^{a}$ & $8.7^{a}$ & $12.4^{\mathrm{b}}$ & 9.5 & 0.002 \\
\hline Experiencia, años & $21.8^{\mathrm{b}}$ & $12.5^{a}$ & $13.7^{a}$ & 17.0 & 0.004 \\
\hline \multicolumn{6}{|l|}{ Comercialización (compra del animal) } \\
\hline Peso vivo del animal en pie, $\mathrm{kg}$ & $40.7^{a}$ & $50.7^{b}$ & $43.8^{a}$ & 45.1 & 0.001 \\
\hline Precio del animal en pie, $\$ / \mathrm{kg}$ & $40.1^{a}$ & $38.7^{a}$ & $43.3^{b}$ & 40.0 & 0.001 \\
\hline \multicolumn{6}{|c|}{ Comercialización (venta del producto procesado) } \\
\hline Precio de venta del producto, $\$ / \mathrm{kg}$ & $324.5^{\mathrm{al}}$ & $281.2^{\mathrm{a} 2}$ & $105.0^{\mathrm{b} 3}$ & 274.9 & 0.001 \\
\hline Venta de producto, kg/semana & $110.7^{a}$ & $48.6^{a}$ & $880.0^{\mathrm{b}}$ & 201.4 & 0.001 \\
\hline
\end{tabular}

Nota: 'Precio del kg de barbacoa. ${ }^{2}$ Precio del kg de barbacoa o birria. ${ }^{3}$ Precio del $\mathrm{kg}$ de carne en canal cruda. Las medias de los grupos con literales diferentes $(a, b)$ difieren estadísticamente $(p<0.05)$. Elaboración propia.

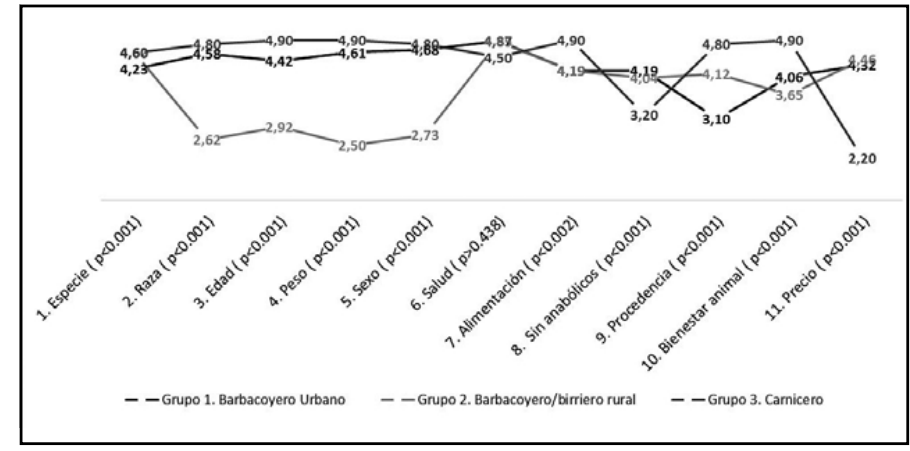

Figura 2. Importancia de los atributos intrínsecos y extrínsecos de los consumidores agroindustriales (escala de 1: Nada importante a 5: Muy importante).

Elaboración propia.

dos con carne tierna y magra (Bernués et al., 2012). Estas características de calidad pueden ser factores afectados, como edad al sacrificio (Barone, Colatruglio, Girolami, Matassino, \& Zullo, 2007), peso vivo (Majdoub-Mathlouthi, Saïd, Say, \& Kraiem, 2013), sexo (Rodrigues \& Teixeira, 2013), y raza (Turner, Belesky, Cassida, \& Zerby, 2014). En este estudio la im- portancia que se dio a los atributos mencionados se debe a que el CAl es quien realizó las compras, procesó y comercializó la carne de los animales, lo que permitió obtener mayor información de su producto.

Este comportamiento puede explicar la preferencia de algunos CAI por animales procedentes de un sistema de alimentación basado en concentrados o pastos y forrajes, atributo que ha sido considerado muy importante por los consumidores finales (Schnettler, Fica, Sepúlveda, Sepúlveda, \& Denegri, 2010), mientras que para otros consumidores es poco importante (Oliazola Tolosana, Whebi, \& Manrique Persiva, 2005). Sin embargo, existen consumidores que dan mayor preferencia a los animales alimentados con pastos o forrajes, o con una mezcla de forraje y concentrado (Font-i-Furnols et al., 2009), con menos residuos de sustancias químicas en la carne (Bernués et al., 2003), y criados bajos condiciones de bienestar animal (Koistinen et al., 2013). Lo anterior también coincide con otros estudios, que han reportado subgrupos de consumidores que difieren en sus 
IIVESTIGACIÓn Y CIERCIA DE LA UNIVERSIDAD AUTÓNOMA

22 apreciaciones sobre los atributos de la carne (Kornelis, van Herpen, van der Lans, \& Aramyan, 2010).

Actualmente, la producción de alimentos de origen animal inocuos, sin residuos químicos, constituye una preocupación importante a escala mundial (Bernués et al., 2003; Santurtún Oliveros, Tapia Pérez, González-Rebeles, \& Galindo Maldonado, 2012). Si bien las posibilidades de intoxicación aguda por los residuos de estas sustancias son bajas (Domínguez-Vara et al., 2009), se desconocen sus efectos en personas que consumen permanentemente estos productos (Márquez Lara, 2008); pocos estudios han abordado este tema en México (Santurtún Oliveros et al., 2012). No obstante, es necesario que el consumidor mexicano esté mejor informado sobre el proceso de producción de carne, que se convierta en un consumidor exigente y adopte hábitos de consumo que no representen riesgos para la salud.

Se presume que el precio es uno de los atributos más importantes en las decisiones de compra; por lo general, se prefieren los precios bajos (Font-i-Furnols et al., 2011), en otros estudios el precio no fue considerado importante en el momento de la compra de la carne (Schnettler et al., 2010). Por otra parte, se menciona que los consumidores se enfocan en aquellos aspectos que permiten optimizar sus recursos económicos (Simons, Francis, Bourlakis, \& Fearne, 2003). En este estudio se encontró que algunos grupos de CAl, a fin de abaratar sus costos de producción, conceden mayor importancia a los precios bajos de animales in vivo. La especie animal es importante para algunos CAl, ya que se relaciona con el tipo de producto terminado (barbacoa o birria) de acuerdo con las costumbres de la región, donde necesariamente buscan ovinos o caprinos que pro- porcionen el gusto del consumidor final (de Andrade, de Aguiar Sobral, Ares, \& Deliza, 2016).

Es evidente que el CAI mexicano de carne ovina y caprina tiene exigencias particulares, que difieren de acuerdo con sus diferentes áreas de influencia y actuación (rural o urbana), que pueden presentar discrepancias en condiciones económicas, educativas, culturales y de salud. El sondeo acerca de producción de carne de pequeños rumiantes en el Estado de México debe orientarse a la búsqueda de estrategias para satisfacer las necesidades del consumidor final e incentivar económicamente a los criadores. La determinación objetiva de la calidad de la canal y carne de los pequeños rumiantes, comercializados en el Estado de México y en el país, permitirá profundizar en las causas y efectos del consumo de carne de pequeños rumiantes, lo que aumentará las posibilidades de mejorar las condiciones de producción y comercialización de carne de ovinos y caprinos.

CONCLUSIONES

Se encontraron tres grupos de consumidores agroindustriales con características particulares que difieren en las exigencias de los atributos de los pequeños rumiantes en cuanto a carne para elaboración de barbacoa, birria, canales o cortes. Los productores de ovinos y caprinos para carne tienen tres opciones para comercializar sus animales de acuerdo con los requerimientos del consumidor agroindustrial.

\section{Agradecimientos}

Los autores desean agradecer a los propietarios de los establecimientos de barbacoa, birria, canales y cortes del Estado de México por toda la información proporcionada para la culminación de este estudio. 


\section{REFERENCIAS}

- Barone, C. M. A., Colatruglio, P., Girolami, A., Matassino, D., \& Zullo, A. (2007). Genetic type, sex, age at slaughter and feeding system effects on carcass and cut composition in lambs. Livestock Science, 112(1-2), 133-142.

- Bernués, A., Olaizola, A., \& Corcoran, K. (2003). Extrinsic attributes of red meat as indicators of quality in Europe: An application for market segmentation. Food Quality and Preference, $14(4), 265-276$.

- Bernués, A., Ripoll, G., \& Panea, B. (2012). Consumer segmentation based on convenience orientation and attitudes towards quality attributes of lamb meat. Food Quality and Preference, 26(2), $211-220$.

- Cochran, W. G. (1984). Técnicas de muestreo. México: CECSA.

- Daniel, W. W. (2005). Bioestadística: Base para el análisis de las ciencias de la salud. (4ª. ed.). México: Limusa Wiley.

- De Andrade, J. C., de Aguiar Sobral, L., Ares, G., \& Deliza, R. (2016). Understanding consumers' perception of lamb meat using free word association. Meat science, 117, 68-74.

- Domínguez-Vara, I. A., Mondragón-Ancelmo, J., GonzálezRonquillo, M., Salazar-García, F., Bórquez-Gastelum, J. L., \& Aragón-Martínez, A. (2009). Los $\beta$-agonistas adrenérgicos como modificadores metabólicos y su efecto en la producción, calidad e inocuidad de la carne de bovinos y ovinos: Una revisión. CIENCIA Ergo-Sum, 16(3), 278-284.

- Font-i-Furnols, M., \& Guerrero, L. (2014). Consumer preference, behavior and perception about meat and meat products: An overview. Meat Science, 98(3), 361-371.

- Font i Furnols, M., Realini, C. E., Guerrero, L., Oliver, M. A., Sañudo, C., Campo, M. M., ... Montossi, F. (2009). Acceptability of lamb fed on pasture, concentrate or combinations of both systems by European consumers. Meat Science, 81 (1), 196-202.

- Font i Furnols, M., Realini, C., Montossi, F., Sañudo, C., Campo, M. M., Oliver, M. A., ...Guerrero, L. (2011). Consumer's purchasing intention for lamb meat affected by country of origin, feeding system and meat price: A conjoint study in Spain, France and United Kingdom. Food Quality and Preference, 22 (5), 443451 .

- Galaviz-Rodríguez, J. R., Vargas-López, S., Zaragoza-Ramírez, J. L., Bustamante González, A., Ramírez-Bribiesca, E., GuerreroRodríguez, J. D., \& Hernández Zapata, J. S. (2011). Evaluación territorial de los sistemas de producción ovina en la región norponiente de Tlaxcala. Revista Mexicana Técnica Pecuaria, 2(1), 53-68.

- Hair, J., Anderson, R., Tatham, R., \& Black, W. (1999). Análisis multivariante ( $5^{a}$. ed). Madrid: Prentice Hall Iberia.
- Juárez-Morales, M., Álvarez-Castañeda, I., Domínguez-Vara, A., Mondragón-Ancelmo, J., Martínez-García, G., BórquezGastélum, J. L., \& Salazar-García, F. (2012). Characterization of farms to identify sheep meat commercialization channels in the highlands of Central Mexico. En Proceedings: 27th World Buiatrics Congress 3-8 june 2012, Lisbon Congress Center, Lisbon Portugal. Lisbonne: Associação Portuguesa de Buiatria.

- Kassa, B. T., Haile, A. G., \& Essa, J. A. (2011). Determinants of sheep prices in the highlands of northeastern Ethiopia: Implication for sheep value chain development. Tropical Animal Health and Production, 43(8), 1525-1533.

- Kinnear, T. C., \& Taylor, J. R. (1993). Investigación de mercados: Un enfoque aplicado (4a. ed.). Santafé de Bogotá: McGrawHill.

- Koistinen, L., Pouta, E., Heikkila, J., Forsman-Hugg, S., Kotro, J., Mäkelä, J., \& Niva, M. (2013). The impact of fat content, production methods and carbon footprint information on consumer preferences for minced meat. Food Quality and Preference, 29(2), 126-136.

- Kornelis, M., van Herpen, E., van der Lans, I., \& Aramyan, L. (2010). Using non-food information to identify food-choice segment membership. Food Quality and Preference, 21 (5), 512520 .

- Majdoub-Mathlouthi, L., Saïd, B., Say, A., \& Kraiem, K. (2013). Effect of concentrate level and slaughter body weight on growth performances, carcass traits and meat quality of Barbarine lambs fed oat hay based diet. Meat Science, 93(3), 557-563.

- Márquez Lara, D. (2008). Residuos químicos en alimentos de origen animal: Problemas y desafíos para la inocuidad alimentaria en Colombia. Corpoica-Ciencia y Tecnología Agropecuaria, 9(1), 124-135.

- Mondragón-Ancelmo, J., Hernández-Martínez, J., RebollarRebollar, S., Salem, A. Z., Rojo-Rubio, R., Domínguez-Vara, I. A., \& García-Martínez, A. (2014). Marketing of meat sheep with intensive finishing in southern state of Mexico. Tropical Animal Health and Production, 46(8), 1427-1433.

- Nam, K. C., Jo, C., \& Lee, M. (2010). Meat products and consumption culture in the East. Meat Science, 86 (1), 95-102.

- Nuncio-Ochoa, G., Nahed, T. J., Díaz Hernández, B., Escobedo Amezcua, F., \& Salvatierra Izaba, E. B. (2001). Caracterización de los sistemas de producción ovina en el estado de Tabasco. Agrociencia, 35(4), 469-477. 
- Oliazola Tolosana, A. M., Whebi, Z., \& Manrique Persiva, E. (2005). Quality perception and consumer attitudes to "specific quality beef" in Aragón, Spain. Spanish Journal of Agricultural Research, 3(4), 418-428.

- Rebollar, S., Hernández Martínez, J., García Salazar, J. A., García-Mata, R., Torres Hernández, G., Bórquez Gastélum, J. L., \& Mejía Hernández, P. (2007). Canales y márgenes de comercialización de caprinos en Tejupilco y Amatepec, Estado de México. Agrociencia, 41 (3), 363-370.

- Rodrigues, S., \& Teixeira, A. (2013). Use of generalized Procrustes analysis (GPA) to test the effects of sex and carcass weight on sensory quality evaluations of Terrincho lamb meat. Meat Science, 93(3), 485-488.

- Santurtún Oliveros, E., Tapia Pérez, G., González-Rebeles, C., \& Galindo Maldonado, F. (2012). Actitudes y percepciones de consumidores en la Ciudad de México, hacia atributos de la producción sustentable de alimentos de origen animal. Veterinaria México, 43(2), 87-101.
- Schnettler, B., Fica, D., Sepúlveda, N., Sepúlveda, J., \& Denegri, M. (2010). Valoración de atributos intrínsecos y extrínsecos en la compra de la carne bovina en el sur de Chile. Revista Científica, 20(1), 101-109.

- Simons, D., Francis, M., Bourlakis, M., \& Fearne, A. (2003). Identifying the determinants of value in the U.K. red meat industry: A value chain analysis approach. Journal on Chain and Network Science, 3(2), 109-121.

- Sistema Integral de Información Agroalimentaria y Pesquera. (s. f.). [Portal electrónico]. Recuperado de http://www.siap. sagarpa.gob.mx/

- Turner, K. E., Belesky, D. P., Cassida, K. A., \& Zerby, H. N. (2014). Carcass merit and meat quality in Suffolk lambs, Katahdin lambs, and meat-goat kids finished on a grass-legume pasture with and without supplementation. Meat Science, 98(2), 211 219. 